\title{
A Topology Discovery Method Based on AFT
}

\author{
Bin Zhang, a , Mei Zhang, b, Yuandong Mao ${ }^{2, b}$, Yun $\mathrm{Yu}^{1, \mathrm{c}}$, Guoquan Jiang ${ }^{1, \mathrm{~d}}$ \\ and Bo Deng ${ }^{1, e}$ \\ ${ }^{1}$ Nanjing Telecommunication Technology Research Institute, Nanjing, China \\ ${ }^{2}$ The First Middle School of Xinxiang Country, Henan, China \\ azhang_bin163@163.com, b657697622@qq.com cyuyun56@163.com, jiianggq2001@163.com, \\ emyhspace@163.com,
}

Keywords: Physical topology discovery; Address forwarding table; Network management

\begin{abstract}
Network physical topology discovery is the key issue for network management and application, the physical topology discovery based on address forwarding table (AFT) is a hot topic on current study. This paper proposes a tree chopping algorithm based on AFT satisfying down constraint, which can discover the physical topology of a subnet accurately.
\end{abstract}

\section{Introduction}

Physical network topology refers to the characterization of the physical connectivity relationships that exist among entities in a network. Many network management tasks rely on knowledge of network physical connectivity. However, obtaining such information is a very difficult task. The complexity of performing Ethernet topology discovery arises from the inherent transparency of Ethernet bridge hardware. Endpoints are unaware of the presence of bridges in the network. The bridges themselves only communicate with their neighbors in the limited exchanges of the spanning tree protocol, and that is not used in all environments.

The only useable MIB information maintained by switches and bridges is in the Address Forwarding Table (AFT) - the set of MAC addresses that are reachable from a port of a given node. If AFTs are complete, (that is, they contain all and only nodes that can be reached from a node's port). However, it is unrealistic to expect that the information in AFT is complete for the real network environment.

In this paper we proposes a tree chopping algorithm based on AFT satisfying down constraint, which can discover the physical topology of a subnet accurately. The proposed algorithm decreases the demand for AFT integrity dramatically, and is the loosest constraint for discovering physical topology which only relies down ports. i.e., If the down ports' AFT of all nodes can define the network physical topology, then the topology can be discovered by the proposed tree chopping algorithm.

\section{Related Work}

The main reason of the hardness of physical topology discovery is: 1) most of the current network topology tools collect and manage networks at the IP layer and require network managers to maintain layer-2 connections manually, furthermore, the layer-2 node's MIB does not provide information on its immediate neighbors. 2) port's AFT provides information of reachable nodes, but the completeness of AFT cannot be guaranteed.

To overcome this difficulty, the IETF has proposed a "physical topology" MIB, but the proposal merely reserves a portion of the MIB space without defining any protocol or algorithm for obtaining the topology information. The work [1] proposed to discover layer-2 topology using port traffic features. Their approach relies on statistical correlation, so it can only infer element connections with some (high) probability; furthermore, it is not at all clear if or how their proposed method would work in the presence of interconnections between network elements belonging to different IP subnets. 
The work [2] proposed a STP based method to discover the connection relationship between physical ports. It can discover all links (not only the active links) between bridges, but it fails to discover the path between bridges and hosts, further the support of STP for all bridges limits its use.

Black et al. [3] listed some problems with finding a layer-2 topology using Bridge MIB data. They proposed a new probing packets based method to find a layer-2 topology without querying network MIB information. However, their approach requires placing custom designed network daemons on each host in the network, which some network managers might find objectionable.

Overall, the above methods have some limitations for discovering layer- 2 topology. AFT based method caused more and more attention recently. The initial algorithm developed by Breitbart et al [4, 5] depended on complete AFT data collected from every single element in the network. Breitbart et al also observed that for multi-subnet networks the network topology may not be unique even for the set of complete AFTs obtained from a simple Ethernet network. Bejerano et al. [6] proposed the first formal algorithm to discover the topology in presence of uncooperative elements (i.e. hubs.) .The algorithm may not discover any topology if the given input set of AFTs defines a non-unique topology. Zheng et al. [7, 8] proposed a method only rely on AFT of down ports of bridges. The method can build connectivity of tree nodes if the down ports' AFT is complete for all nodes.

\section{Topology Discovery Method}

An Ethernet is a graph with two kinds of nodes: hosts, with a single link, and network elements, with multiple links. Thus, we model the network as an undirected topology-tree $G=\langle V ; E\rangle$, where $V$ is a set of all network nodes and each element of $E$ represents a physical connection between two nodes. The network nodes contains bridges (network elements can provide their AFT mibs), hosts (computers and routers, node with multiple network interfaces is treated as multiple hosts), dumb devices (hubs and any network devices which their mibs cannot be accessed). Hence, $\mathrm{V}=\mathrm{S}+\mathrm{H}+\mathrm{D}, \mathrm{S}$ denotes switches or bridges, $\mathrm{H}$ denotes hosts, and $\mathrm{D}$ denotes dumb devices.

Each port of a switch maintains a AFT. We denote the jth interface of a switch Si by Sij. For each interface, the set of addresses that have been learned (by backward learning) on that interface is referred to as the AFT corresponding to Sij and is denoted by Aij. Therefore, Aij is the set of MAC addresses that have been seen as source addresses on packet frames received at Sij.We say that Aij is complete if Aij contains the MAC addresses of all network nodes from which frames can be received at $\mathrm{Sij}$.

Definition 1: If the AFTs of a single subnet can define a unique topology, we call such AFTs satisfy minimal constraint of a single subnet.

Definition 2: Suppose the root switch R is designated, the up port AFT of each switch contains at least one ancestor switch's address (can not be null), if down ports' AFTs of the single subnet can define a unique right topology, we call such down ports' AFTs satisfy minimal down constraint (MDC) of a single subnet.

Definition 3: Suppose the root switch R is designated, the down port AFT of each switch contains at least one descendant's address (can not be null), if up ports' AFTs of the single subnet can define a unique right topology, we call such up ports' AFTs satisfy minimal up constraint of a single subnet.

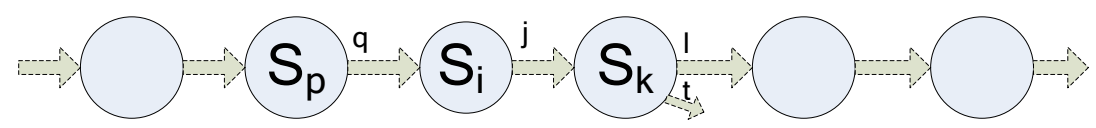

Figure 1. A path in a topology-tree

Lemma 1: For the MDC AFTs, the down port AFT of each node either contains its child's address or contains the child's reachable addresses at least from the child two down ports.

Proof: Fig. 1 illustrates any path in a topology-tree. Si and Sk denote any adjacent nodes, and Si is the father of Sk. We denote Bkt as reachable addresses from down port Skt. The down port constraint AFTs can define the topology solely based on down port AFT. Lemma 1 needs: 1) Sk $\in$ Aij, or 2) if 
Sk $\notin$ Aij, then $\exists \mathrm{a} \in \mathrm{Bkl} \wedge \exists \mathrm{b} \in \mathrm{Bkt}, \quad \partial\{\mathrm{a}, \mathrm{b}\} \subseteq$ Aij. If the two conditions cannot be met, then it must be: 1) Aij is null; or 2) Only exist one port, for example Skl, $\partial(\exists \mathrm{a} \in \mathrm{Bkl} \wedge \mathrm{a} \in \mathrm{Aij})$. In either situation, the node $\mathrm{Si}$ and $\mathrm{Sk}$ can be exchanged in figure 1, which does not influence the down port AFTs of any node in topology-tree. That is, the down port AFTs cannot define a unique topology, which contradicts with the MDC.

Based on the definitions and lemmas in section 3.1, we propose our tree chopping algorithm for MDC AFT as fellows:

Setting up the switch set $\mathrm{H}$ (including the switch address and ports AFT);

Determining all leaf switch Sk: For all switches, select those switches whose only one port AFT (for example Aij) contains other switch address as the primary leaf switch set (lemma 2). Then get rid of mediate switches in the primary set based on lemma 3, we can get the final leaf switch set.

Deleting all leaf switches in $\mathrm{H}$ : Get rid of all leaf switches from $\mathrm{H}$. If a down port AFT of a leaf switch has multiple leaves, the down port connects those leaves with a dumb device.

Updating AFTS in H: Change those addresses in Aij which are contained by the down ports of these cut leaf switches, and merge the repetitive items. This step turns all cut leaf switches into new leafs.

Goto 2), until only the root is left in $\mathrm{H}$.

\section{Evaluation}

We use BRITE and NS2 to evaluate our algorithm. We used BRITEto generate Power-Law-based random tree-like networks with different number of nodes that vary from 50 to 1000 . We use BA model when generating nodes connections by BRTIE. We choose the incremental growth mode and the first node 0 generated as the root.

Our experiments were run on a Pentium 4 2.4GHZ computer with $2 \mathrm{G}$ of RAM and Windows XP operating system. For all the topology generated by BRITE, as long as the constructed AFTs satisfying the MDC, our proposed algorithm is able to correctly determine the topology, which further testifies that a unique topology can be discovered by our proposed algorithm if the AFTs satisfy MDC.

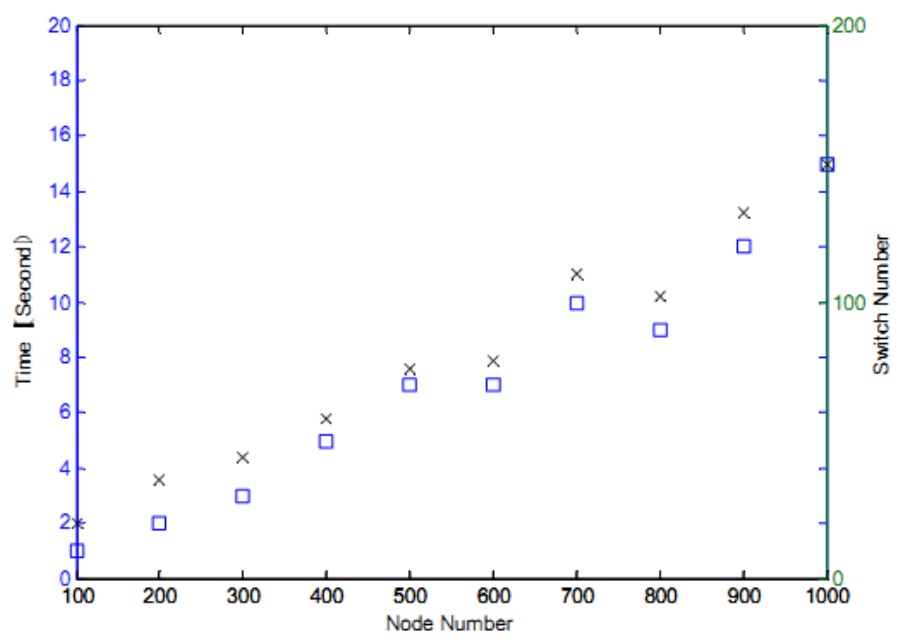

Figure 2. Time to calculate the topology

Although correctness of the topology is the most important criterion when judging the performance of this algorithm, its time performance may also be important in some applications. Fig. 6 shows the time consumed when calculate different nodes topology. The figure is illustrated using a double $\mathrm{Y}$-axis. The $\mathrm{X}$-axis indicates different nodes number of different topologies generated. The Y-axis 1 indicates time used by our algorithm when discovering different nodes topology, which marked with ' $\square$ '. The Y-axis 2 indicates the switches number corresponding to different nodes topology, 
which is marked with ' $\mathrm{X}$ ' . From Fig. 2, we can see that the topology calculating by our algorithm is very fast for a given set of AFTs.

\section{Conclusions}

In this paper, we propose an algorithm which can discover a unique subnet topology based on incomplete AFTs satisfying down constraint. Our proposed algorithm decreases the demand of AFTs integrity dramatically, and can discover a unique topology based on AFTs of down ports meeting MDC. Our proposed algorithm can also be directly used in the switch domain of multiple subnets as long as AFTs of the topology-tree meets lemma 1, and can discover a unique topology of the multiple-subnets switch domain if lemma 1 is met.

\section{Acknowledgements}

This work was supported by the China Postdoctoral Science Foundation under No. 2015M582832, the Jiangsu Post-doctor Research Fund of China under No. 1402138C

\section{References}

[1] Dawes N, Schenkel D, Slavitch M. Method of determining the topology of a network of objects. U.S. Patent 6,231,997, June 7, 2002.

[2] Son MH, Joo BS, Kim BC, Lee JY. Physical topology discovery for metro ethernet networks. ETRI Journal, 2005, 9(4):175-186.

[3] Black R, Donnelly A, Fournet C. Ethernet topology discovery without network assistance. In: La Porta T, Ramjee R, Koenig H, Effelsberg W, eds. Proc. of the 12th IEEE Int'l Conf. on Network Protocols (ICNP 204). Los Alamitos: IEEE Computer Society, 2004. 130-159.

[4] Breitbart Y, Garofalakis M, Martin C, Rastogi R, Seshadri S, Silberschatz A. Topology discovery in heterogeneous IP networks. In: Sidi M, Sengupta B, eds. Proc. of the INFOCOM 2000. New York: IEEE Press, 2000. 85-94.

[5] Breitbart Y, Garofalakis M, Jai B, Martin C, Rastogi R, Silberschatz A. Topology discovery in heterogeneous IP networks: The NetInventory system. IEEE/ACM Trans. on Networking, 2014, 12 (3):221-234.

[6] Bejerano Y, Breitbart Y, Garofalakis M, Rastogi R. Physical topology discovery for large multi-subnet networks. In: Bauer F, Roberts J, Shroff N, eds. Proc. of the IEEE INFOCOM 203. New York: IEEE Press, 2003. 162-172.

[7] Zheng H, Zhang GQ. An algorithm for physical network topology discovery. Journal of Computer Research and Development, 2002, 39 (3):264-268 (in Chinese with English abstract).

[8] Fu C,Jiahai Y,Yang Y. New Algorithms on IP Network Topology Discovery and Its Implement. ACTA ELECTRONICA SINICA, Aug. 2008, 36(8):1620-1625. 